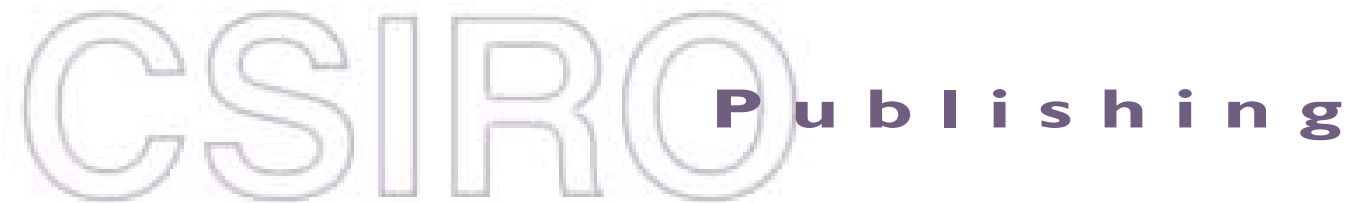

\section{Publications of the Astronomical Society of Australia}

Volume 19, 2002

(C) Astronomical Society of Australia 2002

An international journal of astronomy and astrophysics

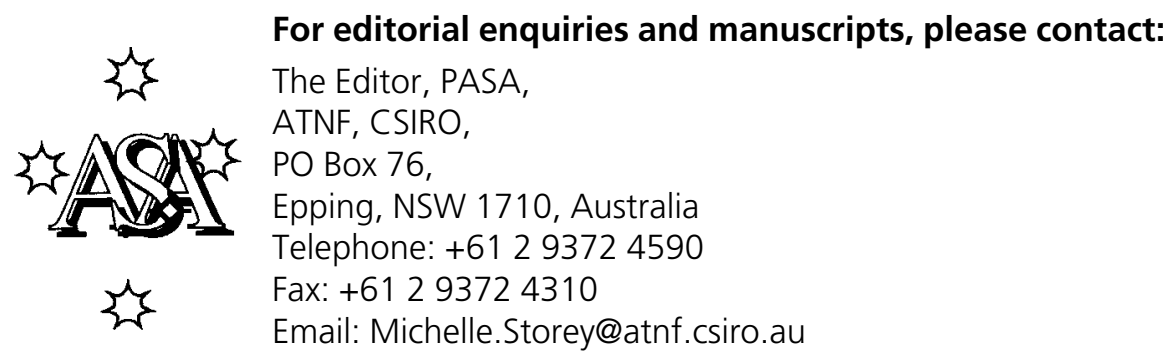

For general enquiries and subscriptions, please contact: CSIRO Publishing PO Box 1139 (150 Oxford St)

Collingwood, Vic. 3066, Australia

Telephone: +6139662 7666

Fax: +61 396627555

Email: publishing.pasa@csiro.au

C S I RO

PUBLISHING Published by CSIRO Publishing

for the Astronomical Society of Australia

www.publish.csiro.au/journals/pasa 


\title{
A Spectral Line Survey of IRAS 17470-2853 from 86.1 to $92.1 \mathrm{GHz}$
}

\author{
Hun-Dae Kim ${ }^{1}$, Ramesh Balasubramanyam ${ }^{1}$ and Michael G. Burton ${ }^{1,2}$ \\ ${ }^{1}$ School of Physics, University of New South Wales, Sydney, NSW 2052, Australia \\ ${ }^{2}$ School of Cosmic Physics, Dublin Institute for Advanced Studies, 5 Merrion Square, Dublin 2, Ireland
}

Received 2001 September 15, accepted 2002 October 6

\begin{abstract}
We present results from a spectral line survey of the young stellar object IRAS 17470-2853, undertaken to examine chemical changes during the evolution from hot molecular cores to ultracompact $\mathrm{HII}$ regions. Observations were carried out with the Mopra $22 \mathrm{~m}$ radio telescope in the frequency range from 86.1 to $92.1 \mathrm{GHz}$. A total of 21 lines from 9 molecules were detected. Except for $\mathrm{CH}_{3} \mathrm{CN}$ they are all simple molecules. We compare the results to the ultracompact HII region G34.3+0.15, where spectral line surveys in the frequency range $80-115 \mathrm{GHz}$ and $330-360 \mathrm{GHz}$ have been performed. While the molecular lines detected are similar, their widths and intensities are somewhat narrower and lower, respectively, in IRAS 17470-2853. The typical line width of $\sim 5 \mathrm{~km} \mathrm{~s}^{-1}$ indicates relatively quiet or quasi-thermal emission. On the other hand, a significant difference in $T_{A}^{*}(\mathrm{HNC}) / T_{A}^{*}(\mathrm{HCN})$ has been found: 0.8 for IRAS 17470-2853 compared to 2.6 for $\mathrm{G} 34.3+0.15$. The broad line width of $\mathrm{SiO}(v=0, J=2-1), \sim 9 \mathrm{~km} \mathrm{~s}^{-1}$, suggests that IRAS 17470-2853 is experiencing a shock generated by the embedded object. Column densities, or lower limits to them, are derived for observed molecules.
\end{abstract}

Keywords: HII regions — ISM: individual (IRAS 17470-2853 = G0.55-0.85) — ISM: molecules — ISM: abundances — stars: formation — radio lines: ISM

\section{Introduction}

Understanding the relation between the stage that star formation has reached and the chemical state of a molecular cloud has long been of interest, since young stellar objects (YSOs) drive the chemical evolution of molecular clouds, especially adjacent to a hot core. A hot core is characterised by small volume, high density, and warm temperature (typically $R \sim 0.01-0.1 \mathrm{pc}, n \sim 10^{6}$ $10^{8} \mathrm{~cm}^{-3}, T \sim 100-300 \mathrm{~K}$ : Henkel et al. 1987; Walmsley et al. 1987), and is associated with an embedded protostar. Hot cores are also distinguished from cold cores through their high abundance of saturated molecules such as $\mathrm{CH}_{3} \mathrm{OH}, \mathrm{NH}_{3}, \mathrm{CH}_{3} \mathrm{CN}, \mathrm{CH}_{3} \mathrm{CH}_{2} \mathrm{OH}$, and $\mathrm{H}_{2} \mathrm{CS}$. The high abundance of such complex molecules cannot be explained solely by pure gas phase reactions in the cloud, but requires grain surface chemistry. Even though the details of how the chemistry works remain controversial, the models (e.g., Millar, Herbst, \& Charnley 1991; Charnley et al. 1995) produce reasonably close agreement with observational results. In grain surface models the high abundance of complex molecules is due to hydrogenation and nitrogenation of atoms and molecules that are deposited onto the dust mantles as the molecular clouds undergo gravitational collapse. As star formation commences, radiation from the embedded protostar evaporates the molecules from the dust mantles. Accordingly, star formation determines the overall chemical reactions and evolution of the parent molecular clouds.

Through an understanding of these chemical properties we can trace the evolutionary phase of molecular clouds by comparing chemical models to observation. Star-forming clouds for which chemical models have been developed include Orion-KL (Charnley et al. 1995) and G34.3+0.15
(Millar, Macdonald, \& Gibb 1997). The models are reasonably successful, but they require the inclusion of more complicated reaction routes, detailed calculations of collision rates, and improvements to their time-dependent behaviour to provide a more precise explanation for the observational data. Molecules such as $\mathrm{CH}_{3} \mathrm{CN}, \mathrm{CH}_{3} \mathrm{OH}$, and $\mathrm{NH}_{3}$, believed to probe the hot cores, have been widely used to delineate their chemical properties. However, they are not enough to derive the overall state of star-forming molecular clouds, which comprise a range of temperatures and densities from cold gas to hot cores. Changes occurring within the hot core then influence the chemistry within the ambient molecular cloud, through both the radiation field and outflows that are generated. Hence, a study of both the cold gas and hot core region of clouds is required to provide a more comprehensive understanding of the effects of star formation on and in molecular clouds.

Molecular line surveys satisfy these requirements. Their wide frequency range includes emission from many species and their transitions have a wide range of excitation energy. Comparison of detected molecules and their transitions between sources allows us to examine the variation in chemical properties from source to source. If many transitions from a molecule are detected, it is possible to estimate excitation temperatures and molecular abundances by means of the rotation diagram method (e.g., see Macdonald et al. 1996). While extensive observations have been carried out on the sources OrionKL (e.g., Johansson et al. 1984; Jewell et al. 1989; Turner 1989; Blake et al. 1986, 1996; Ziurys \& McGonagle 1993; Schilke et al. 1997) and Sgr B2 (e.g., Cumins, Linke, \& Thaddeus 1986; Turner 1989; Sutton et al. 1991), a wider range of sources is needed in order to investigate 
the chemical evolution underway. Therefore, we undertook a molecular line survey of the young stellar source IRAS $17470-2853$ to examine its physical and chemical properties. We have compared these to the ultracompact HII region $\mathrm{G} 34.3+0.15$, because $\mathrm{G} 34.3+0.15$ seems to be more alike in evolutionary stage and physical properties than Orion-KL or Sgr B2 are.

Walsh et al. (1997, 1998) undertook a survey of ultracompact HII regions for emission from the $6.669 \mathrm{GHz}$ methanol maser, to examine the association between these two signposts of star formation. Among their sources IRAS 17470-2853 (also known as G0.55-0.85) was included. Its distance is inferred to be $2 \mathrm{kpc}$ and the radial velocity range of the methanol masers is +8 to $+19 \mathrm{~km} \mathrm{~s}^{-1}$. Also measured from this source is radio and sub-mm continuum emission, $\mathrm{OH}$ and water maser emission (see Walsh et al. 1998, 1998, 2002; Forster \& Caswell 1999). The $\mathrm{OH}$ masers have similar velocity range to the methanol masers $\left(+8\right.$ to $\left.+20 \mathrm{~km} \mathrm{~s}^{-1}\right)$, but the water masers are spread over a larger velocity range, from -13 to $+57 \mathrm{~km} \mathrm{~s}^{-1}$. The radio and sub-mm continuum emission consists of a bright core, embedded in diffuse emission extending mostly NW-SE of it. The methanol and $\mathrm{OH}$ maser sites also are coincident with the continuum core, and the $\mathrm{OH}$ maser sites appear to extend somewhat in a direction NW-SE. The water maser sites are located $\sim 2^{\prime \prime} \mathrm{NW}$ of the $\mathrm{OH}$ and $\mathrm{CH}_{3} \mathrm{OH}$ masers. The source also appears in the $\mathrm{MSX}^{1}$ survey at mid-infrared wavelengths, as one of six sources embedded in diffuse emission over a $30^{\prime}$ region. It is also visible as a faint red source in the 2MASS near-infrared survey, with a $[1.65 \mu \mathrm{m}]-[2.2 \mu \mathrm{m}]$ colour of 2.4 magnitudes. The bolometric luminosity of the source has been estimated as $\sim 1.3 \times 10^{4} \mathrm{~L}_{\odot}$ (Walsh et al. 2002), the equivalent of a B0.5 main sequence star. For reference, the fluxes from near-infrared to radio wavelengths are tabulated in Table 1. To aid identification of the features, Figure 1, adapted from Walsh et al. (2002), shows the sub-mm continuum emission, and the positions of the methanol masers, radio continuum emission, and sources from the MSX point source catalogue.

From their survey Walsh et al. (1998) concluded that methanol maser emission arises before the onset of ultracompact HII region formation, and disappears sometime after its expansion towards an HII region. The detection of methanol maser emission with relatively weak radio continuum from IRAS 17470-2853 suggests that it is probably less evolved than G34.3+0.15. Accordingly, our investigation with the Mopra telescope sought to examine whether the chemical state of the source supported this supposition that it is in an earlier evolutionary stage.

\section{Observations}

Observations of IRAS 17470-2853 in the frequency range $86.1-92.1 \mathrm{GHz}$ were made using the Mopra $22 \mathrm{~m}$ radio

${ }^{1}$ For MSX and 2MASS infrared survey archives see http://irsa.ipac. caltech.edu.
Table 1. Continuum fluxes of IRAS 17470-2853 (G0.55-0.85)

\begin{tabular}{lc}
\hline $\begin{array}{l}\text { Wavelength } \\
(\mu \mathrm{m})\end{array}$ & $\begin{array}{c}\text { Flux } \\
(\mathrm{Jy})\end{array}$ \\
\hline $1.25^{1}$ & $2.0(-4)$ \\
$1.65^{1}$ & $1.2(-3)$ \\
$2.2^{1}$ & $6.8(-3)$ \\
$8.3^{2}$ & $1.6(+1)$ \\
$12.1^{2}$ & $2.7(+1)$ \\
$14.6^{2}$ & $3.3(+1)$ \\
$21.3^{2}$ & $1.4(+2)$ \\
$450^{3}$ & $4.4(+2)$ \\
$850^{3}$ & $4.7(+1)$ \\
$34700^{4,5}$ & $7.0(-2)$ \\
\hline
\end{tabular}

In the Flux column $2.0(-4) \equiv 2.0 \times 10^{-4}$, etc.

${ }^{1}$ From 2MASS archive for source at $17^{h} 50^{m} 14.4^{s}$,

$-28^{\circ} 54^{\prime} 26^{\prime \prime}$ (J2000); ${ }^{2}$ From MSX archive for source at $17^{h} 50^{m} 14.4^{s},-28^{\circ} 54^{\prime} 28^{\prime \prime}(\mathrm{J} 2000){ }^{3}$ From Walsh et al. (2002) for source at $17^{h} 50^{m} 14.2^{s},-28^{\circ} 54^{\prime} 30^{\prime \prime}(\mathrm{J} 2000)$;

${ }^{4}$ From Walsh et al. (1998) for source at $17^{h} 50^{m} 14.5^{s}$,

$-28^{\circ} 54^{\prime} 30^{\prime \prime}(\mathrm{J} 2000) ;{ }^{5}$ More usually written as $8.64 \mathrm{GHz}$.

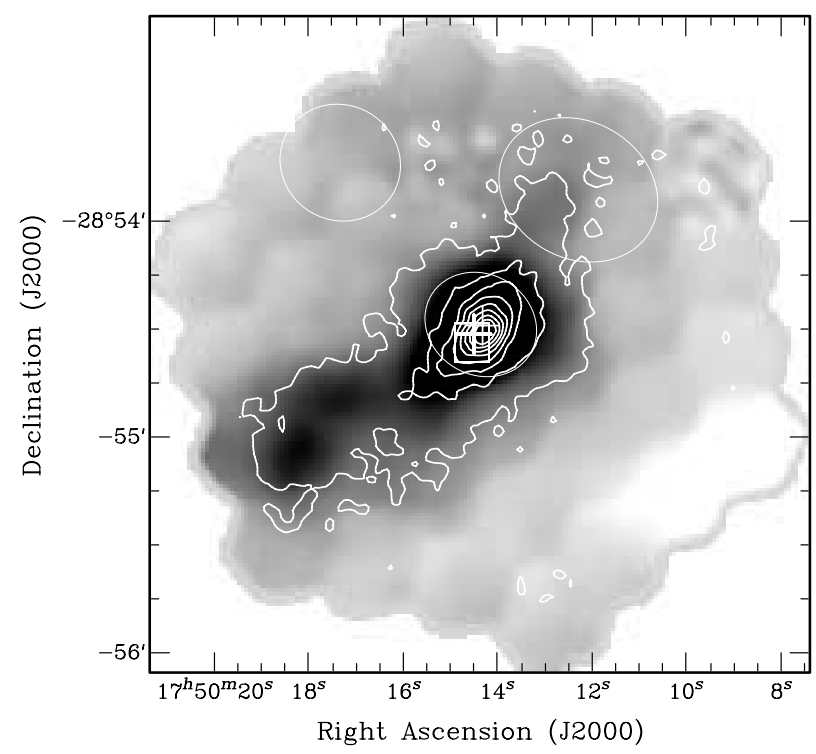

Figure 1 Image of the $850 \mu \mathrm{m}$ emission from IRAS 17470-2853, overlaid with contours of the $450 \mu \mathrm{m}$ emission (adapted from Walsh et al. 2002). Also shown with crosses are the positions of methanol maser sites, with a square the position of the radio continuum emission from an ultracompact HII region, and with ellipses the positions of MSX sources from the Point Source Catalogue (version 1.2).

telescope between 20 and 30 November 2000. The position observed was $17^{h} 50^{m} 14.5^{s},-28^{\circ} 54^{\prime} 31.2^{\prime \prime}$ (J2000), with a sky position $+1^{m}$ away in longitude. The beam size is $40^{\prime \prime}$ and the forward spillover and scattering efficiency $\left(\eta_{\mathrm{fss}}\right)$ of the telescope is 0.6 . The receiver employed was a dual polarisation, low noise SiS mixer, with an autocorrelator configured to have 512 channels of $500 \mathrm{kHz}$ width each $\left(\sim 1.5 \mathrm{~km} \mathrm{~s}^{-1}\right)$. This allowed $256 \mathrm{MHz}$ coverage for each local oscillator (LO) setting. In the course of 
observations the typical system temperature ranged from 200 to $400 \mathrm{~K}$. Observations were carried out in positionswitched mode, with $30 \mathrm{~s}$ on-source and $30 \mathrm{~s}$ off-source, repeating typically 20 times. The same LO frequency was set for both polarisation $\mathrm{A}$ and $\mathrm{B}$, improving the RMS noise by a factor of $\sqrt{2}$. The system provides a theoretical RMS noise of 0.03-0.04 K, allowing the identification of lines with antenna temperature $T_{A}^{*}$ of $\sim 0.1 \mathrm{~K}(3 \sigma)$. Pointing was checked every $2-3 \mathrm{~h}$, targeting an $\mathrm{SiO}$ $86 \mathrm{GHz}$ maser point source, $\mathrm{VX}-\mathrm{Sgr}$.

With some considerable tuning and pointing overheads, we obtained 3-4 spectra of $256 \mathrm{MHz}$ bandwidth during each $6 \mathrm{~h}$ observing session. Flux calibration was done with standard chopper wheel method (Ulich \& Haas 1976), giving the intensity on the antenna temperature scale, $T_{A}^{*}$.

\section{Spectral Reduction and Line Identification}

Reduction of the spectral scans was undertaken with the AIPS++ DISH tool (Garwood \& McMullin 1999), together with custom-written auxiliary programs. In averaging spectra from individual scans, those with poor baselines were first discarded, a 3rd order polynomial fit to the continuum and the baseline then subtracted off. The typical RMS noise achieved per spectrum was $0.05 \mathrm{~K}$, about a factor 2 worse than theoretically possible if no bad scans were thrown out. The signal at the edge of the bandpass was usually poor and so discarded, leading to some small gaps in spectral coverage. The spectra from each resulting $\sim 200 \mathrm{MHz}$ band were then combined with neighbouring bands, so each panel presented in Figure 2 covers about $800 \mathrm{MHz}$. For line identification, we used the Lovas catalogue (Lovas 1992) exclusively, because the frequency $86-92 \mathrm{GHz}$ has been fully covered in other line surveys.

Central frequencies for each line stronger than $3 \sigma$ were determined through Gaussian fitting, after correcting for the Doppler shift of $+14 \mathrm{~km} \mathrm{~s}^{-1}$. A total of 21 lines, including blended lines, from 9 species were detected (see Table 2). All the lines have also been detected in G34.3+0.15 (Kim et al. 2000). There are also some notable differences, however, from G34.3+0.15, as discussed in Section 4. As anticipated, the spectrum is somewhat different from that of Orion-KL (Turner 1989), reflecting the different chemical state of the sources.

\section{Individual Molecules}

In Figure 3 we compare the measured values of $T_{A}^{*}$, FWHM, and intensity $\left(\int T_{A}^{*} d v\right)$ for IRAS 17470-2853 with those for G34.3+0.15 obtained with the $14 \mathrm{~m}$ Taeduk Radio Astronomy Observatory (TRAO) telescope. This figure demonstrates graphically the difference in physical parameters derived for molecules in the two sources, although the different beam sizes render the Mopra results more sensitive to compact (i.e. unresolved) emission. In general the intensities, $T_{A}^{*}$, are similar, except for $\mathrm{HCN}$ which is four times brighter in IRAS 17470-2853. The typical line width in IRAS $17470-2853, \sim 5 \mathrm{~km} \mathrm{~s}^{-1}$, is somewhat narrower than $\mathrm{G} 34.3+0.15$. The $\mathrm{SiO}(v=0$, $J=2-1)$ line has a broader line width $\left(9.1 \mathrm{~km} \mathrm{~s}^{-1}\right)$, although it is also slightly narrower than in G34.3+0.15 (where it is $10.0 \mathrm{~km} \mathrm{~s}^{-1}$ wide).
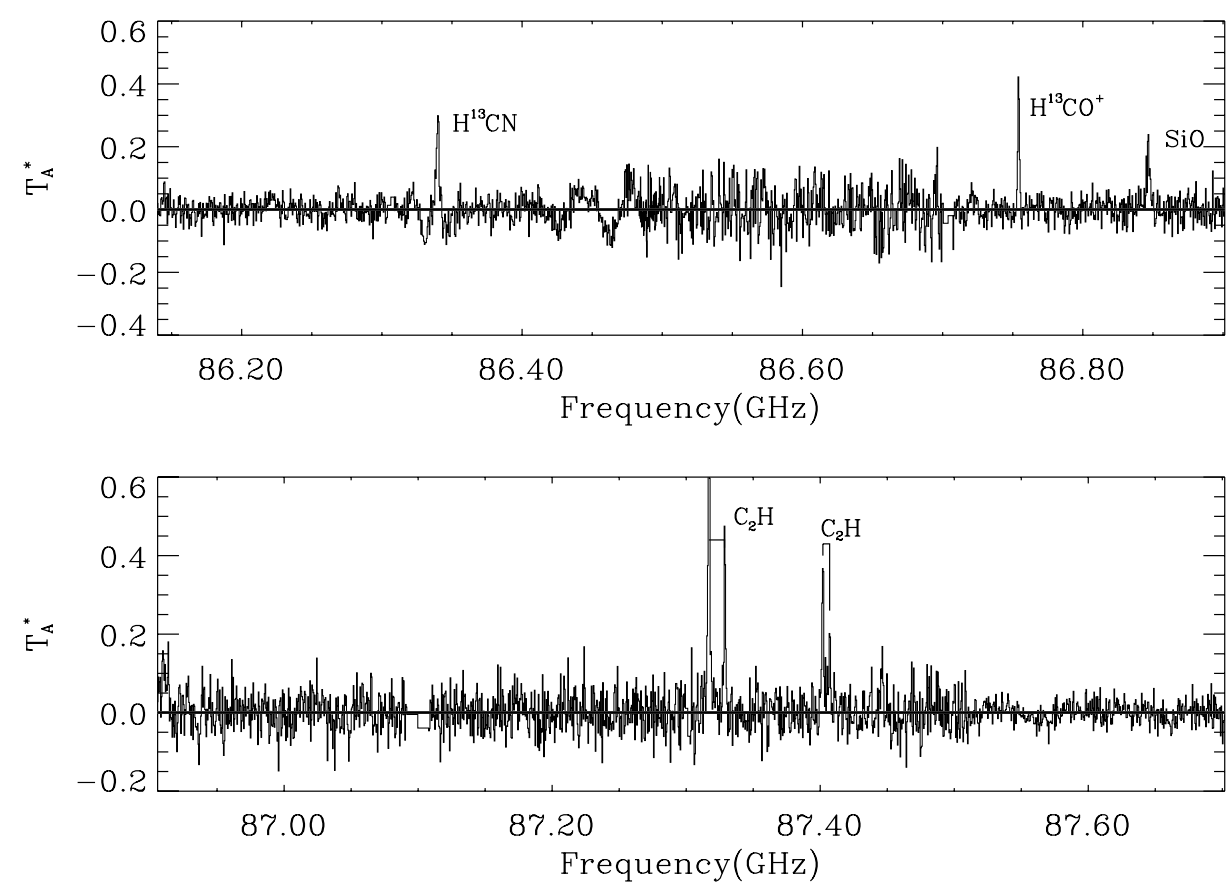

Figure 2 Spectra of the 86.1-92.1 GHz line emission from IRAS 17470-2853. Each panel is $800 \mathrm{MHz}$ wide, combining four neighbouring frequency bands. Detected lines are labelled with their molecule. The intensity scale is $T_{A}^{*}$ and the frequency scale is in GHz. 

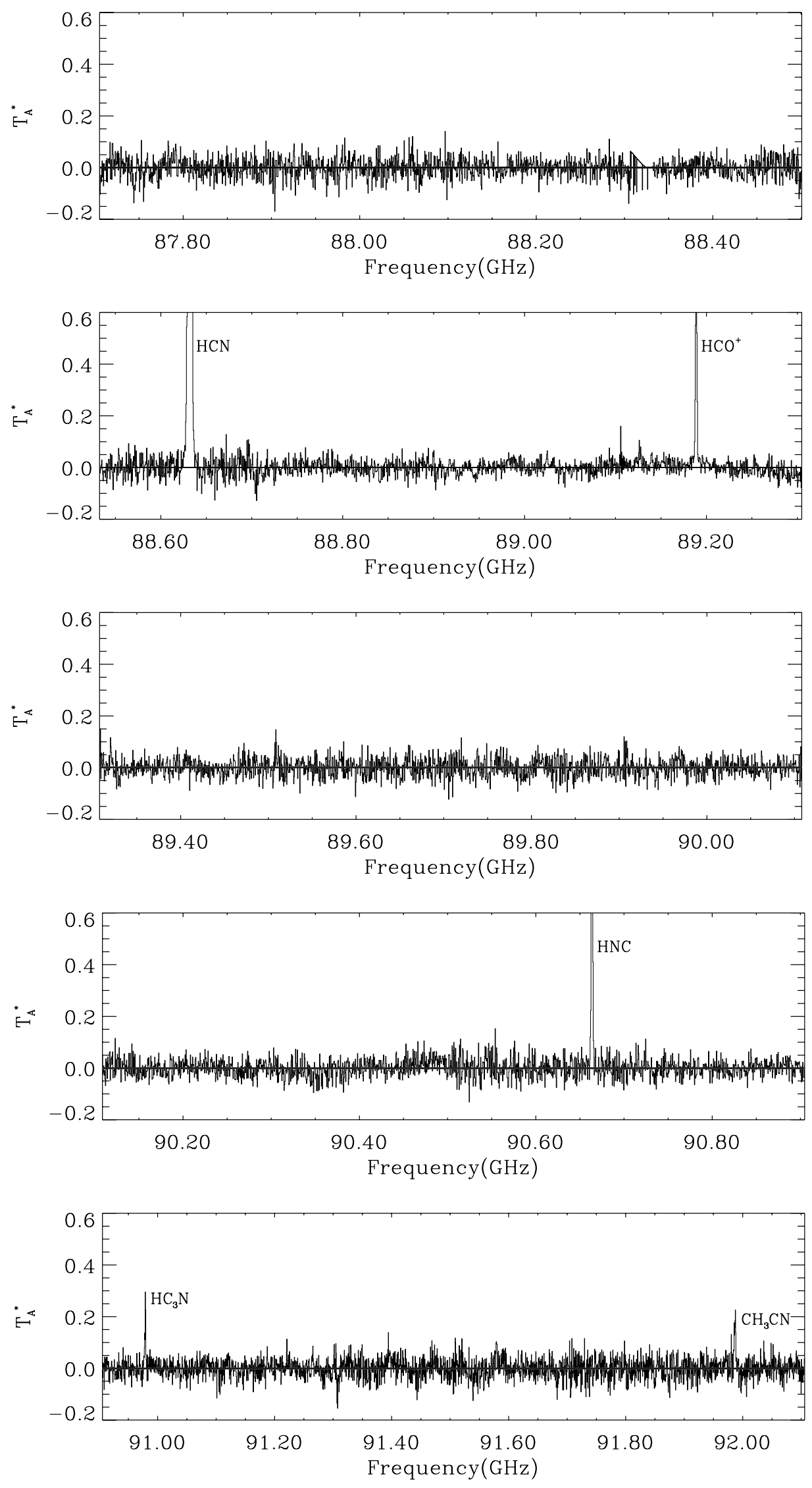

Figure 2 (continued) 
Table 2. Observed molecular line parameters ${ }^{a}$

\begin{tabular}{|c|c|c|c|c|c|c|c|}
\hline $\begin{array}{l}v(\mathrm{obs})^{b} \\
(\mathrm{GHz})\end{array}$ & $\begin{array}{l}T_{A}^{*} \\
(\mathrm{~K})\end{array}$ & $\begin{array}{c}\Delta v \\
\left(\mathrm{~km} \mathrm{~s}^{-1}\right)\end{array}$ & Species & Transition & $\begin{array}{l}v \text { (rest) } \\
(\mathrm{GHz})\end{array}$ & $\begin{array}{c}\int T_{A}^{*} d \nu^{c} \\
\left(\mathrm{Kkm} \mathrm{s}^{-1}\right)\end{array}$ & Notes \\
\hline \multirow[t]{3}{*}{86.3398} & \multirow[t]{3}{*}{$0.3^{*}$} & \multirow[t]{3}{*}{8.6} & $\mathrm{H}^{13} \mathrm{CN}$ & $1-0 F=1-1$ & 86.3388 & \multirow[t]{3}{*}{2.6} & bld \\
\hline & & & $\mathrm{H}^{13} \mathrm{CN}$ & $1-0 F=2-1$ & 86.3402 & & bld \\
\hline & & & $\mathrm{H}^{13} \mathrm{CN}$ & $1-0 F=0-1$ & 86.3423 & & \\
\hline 86.7542 & 0.4 & 4.7 & $\mathrm{H}^{13} \mathrm{CO}^{+}$ & $1-0$ & 86.7543 & 2.2 & \\
\hline 86.8467 & 0.2 & 9.1 & $\mathrm{SiO}$ & $2-1 v=0$ & 86.8470 & 1.7 & \\
\hline 87.3170 & 0.8 & 5.1 & $\mathrm{C}_{2} \mathrm{H}$ & $1-03 / 2-1 / 2 F=2-1$ & 87.3169 & 4.4 & \\
\hline 87.3287 & 0.5 & 4.8 & $\mathrm{C}_{2} \mathrm{H}$ & $1-03 / 2-1 / 2 \quad F=1-0$ & 87.3286 & 2.0 & \\
\hline 87.4019 & 0.4 & 5.2 & $\mathrm{C}_{2} \mathrm{H}$ & $1-01 / 2-1 / 2 F=1-1$ & 87.4020 & 2.1 & \\
\hline 87.4071 & 0.2 & 3.6 & $\mathrm{C}_{2} \mathrm{H}$ & $1-01 / 2-1 / 2 F=0-1$ & 87.4072 & 0.8 & \\
\hline 87.4461 & 0.1 & 6.0 & $\mathrm{C}_{2} \mathrm{H}$ & $1-01 / 2-1 / 2 F=1-0$ & 87.4465 & 0.9 & \\
\hline \multirow{3}{*}{88.6318} & \multirow{3}{*}{$2.9^{*}$} & \multirow[t]{3}{*}{15.9} & $\mathrm{HCN}$ & $1-0 F=1-1$ & 88.6304 & \multirow{3}{*}{36.9} & bld \\
\hline & & & $\mathrm{HCN}$ & $1-0 F=2-1$ & 88.6318 & & bld \\
\hline & & & $\mathrm{HCN}$ & $1-0 F=0-1$ & 88.6339 & & bld \\
\hline 89.1887 & 1.4 & 4.5 & $\mathrm{HCO}^{+}$ & $1-0$ & 89.1885 & 6.5 & \\
\hline \multirow[t]{3}{*}{90.6635} & \multirow[t]{3}{*}{$2.4^{*}$} & \multirow[t]{3}{*}{4.2} & $\mathrm{HNC}$ & $1-0 F=0-1$ & 90.6635 & \multirow[t]{3}{*}{11.7} & bld \\
\hline & & & HNC & $1-0 F=2-1$ & 90.6636 & & bld \\
\hline & & & $\mathrm{HNC}$ & $1-0 F=1-1$ & 90.6637 & & bld \\
\hline 90.9790 & 0.3 & 5.8 & $\mathrm{HC}_{3} \mathrm{~N}$ & $10-9$ & 90.9790 & 1.5 & \\
\hline 91.9806 & 0.1 & 2.9 & $\mathrm{CH}_{3} \mathrm{CN}$ & $J=5-4, K=2$ & 91.9801 & 0.4 & \\
\hline \multirow[t]{2}{*}{91.9868} & \multirow[t]{2}{*}{$0.2^{*}$} & \multirow[t]{2}{*}{10.5} & $\mathrm{CH}_{3} \mathrm{CN}$ & $J=5-4, K=1$ & 91.9853 & \multirow[t]{2}{*}{2.1} & bld \\
\hline & & & $\mathrm{CH}_{3} \mathrm{CN}$ & $J=5-4, K=0$ & 91.9871 & & bld \\
\hline
\end{tabular}

${ }^{a}$ Molecules and their transitions detected in the frequency range of 86.1-92.1 GHz. Blended lines are marked with 'bld' in the notes column. The flux listed is for the sum of all lines in the blend.

${ }^{b}$ Observed frequencies after correcting the Doppler shift with the velocity of the local standard of rest, $+14 \mathrm{~km} \mathrm{~s}^{-1}$ (Walsh et al. 1997). The full-width half-maximum (FWHM) and antenna temperature $\left(T_{A}^{*}\right)$ of each line are derived from Gaussian fitting, except for $T_{A}^{*}$ for $\mathrm{HNC}$ and $\mathrm{HCN}$, where the peak value of the signal is used.

${ }^{c *}$ in the $T_{A}^{*}$ column refers to the peak value of the signal in blended lines.

\section{1 $\mathrm{HCO}^{+}, \mathrm{HCN}$, and $\mathrm{HNC}$}

$\mathrm{HCO}^{+}, \mathrm{HCN}$, and $\mathrm{HNC}$ are all detected in IRAS 174702853. The isotopes $\mathrm{H}^{13} \mathrm{CN}$ and $\mathrm{H}^{13} \mathrm{CO}^{+}$are also detected, and the relevant isotopic line ratios are $\mathrm{HCN} / \mathrm{H}^{13} \mathrm{CN} \sim 9$ and $\mathrm{HCO}^{+} / \mathrm{H}^{13} \mathrm{CO}^{+} \sim 3$.

\section{$\mathrm{HCO}^{+}$}

Figure 4 compares the $\mathrm{HCN}$ and $\mathrm{HCO}^{+}$profiles in IRAS $17470-2853$ and G34.3+0.15. No self-absorption feature is seen in the $\mathrm{HCO}^{+}(1-0)$ line in IRAS 174702853 , unlike in G34.3+0.15, where there is a dip in the profile at $+3 \mathrm{~km} \mathrm{~s}^{-1}$. This suggests that IRAS 17470 2853 is surrounded by a relatively thinner colder layer than $\mathrm{G} 34.3+0.15$, so the emission passes through the foreground cloud without absorption.

\section{$H C N$}

When optically thin, the ratio of the $F=1-1,2-1$ and $0-1$ hyperfine transitions of $\mathrm{HCN}$ is 3:5:1, i.e. the ratio of the level degeneracies. While the 2-1 and 0-1 lines are blended in our data, the observed ratio in IRAS 174702853 is clearly $\sim 3: 5: 3$ rather than 3:5:1. These ratios are very different, however, to those measured in $\mathrm{G} 34.3+0.15$ (which are $\sim 6: 5: 2$; see Figure 4), which we attribute to optical depth effects in the 2-1 and 1-1 lines in that source. For instance, Cernicharo et al. (1984) suggest that such anomalous ratios can arise due to the scattering of these two lines by a diffuse envelope surrounding an optically thick hotter core, while leaving the optically thin 0-1 line unattenuated.

\section{$H N C$}

The isomers $\mathrm{HCN}$ and $\mathrm{HNC}$ have a similar energy level structure and almost identical dipole moments: $\mu=2.98$ debyes for $\mathrm{HCN}$ and 3.05 for HNC. They also have the same pre-cursor molecules, $\mathrm{HCNH}^{+}$and $\mathrm{H}_{2} \mathrm{CN}^{+}$(Brown, Burden, \& Cuno 1989). Therefore one expects a similar abundance ratio for the two isomers. However, the ratio $T_{A}^{*}(\mathrm{HNC}) / T_{A}^{*}(\mathrm{HCN})$ is very different between the sources: 0.8 in IRAS $17470-2853$ and 2.6 in G34.3+0.15. The higher ratio in G34.3+0.15 might arise from selfabsorption in the HCN line, as discussed above. However, if self-absorption is not significant for the HCN line, then the difference in $\mathrm{HNC} / \mathrm{HCN}$ ratios may mark a physical difference between the environment in the two sources (e.g., see Schilke et al. 1992), with a greater abundance of HNC in IRAS 17470-2853. If so, then measurement of this line ratio provides a tool to study the change of physical state in evolving hot molecular cores.

It would, however, be unusual to have a greater abundance of HNC than HCN. For instance, in a study of molecular abundances in 13 molecular clouds Wootten et al. (1978) found that in most sources the abundance ratio of $\mathrm{HNC} / \mathrm{HCN}$ is less than 1. Baudry et al. (1980) also found in a study towards 9 molecular clouds that HNC 

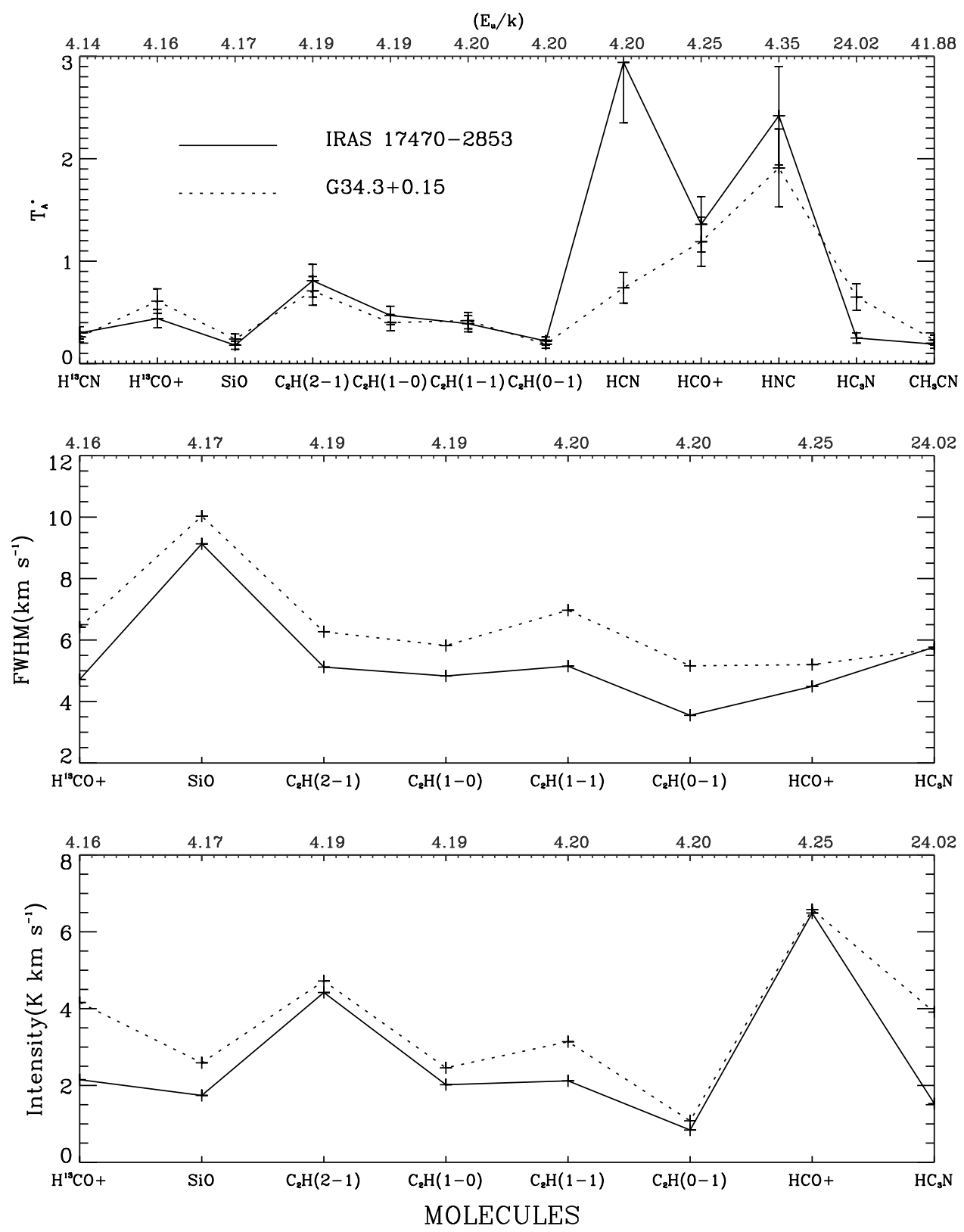

Figure 3 A comparison of $T_{A}^{*}$, line FWHM (in $\mathrm{km} \mathrm{s}^{-1}$ ), and line intensity (in $\mathrm{K} \mathrm{km} \mathrm{s}^{-1}$ ) between the different molecules detected by us in IRAS 17470-2853 (solid line) to G34.3+0.15 (dashed line, obtained with the $14 \mathrm{~m}$ TRAO telescope by Kim et al. 2000). $T_{A}^{*}$ is shown for all lines, whereas the FWHM and line intensity are only shown for lines which are not blended. The upper horizontal axis indicates the energy level of the upper state of each transition. Errors bars show a $20 \%$ error determined for absolute calibration.

is not more abundant than HCN. Goldsmith et al. (1981) similarly found $\mathrm{HCN}$ to be more abundant in giant molecular clouds with active star formation, such as W51 and Orion. On the other hand, in quiescent dark clouds Irvine $\&$ Schloerb (1984) found HNC to be more abundant than $\mathrm{HCN}$, as did Huttemeister et al. (1995) in the extragalactic source Arp 220, a result attributed by the latter authors to emission from a cool, quiescent disk or halo around the source. Thus, the observations of HCN and HNC empirically support IRAS $17470-2853$ being warmer than the cold dark clouds.

Finally, we note that $\mathrm{HN}^{13} \mathrm{C}$ is not detected at $87.09 \mathrm{GHz}$ (Figure 2), whereas it is seen in G34.3+0.15
(Kim et al. 2000). However, this is most likely due to a gap in the spectral coverage at this frequency.

\section{$4.2 \mathrm{C}_{2} \mathrm{H}$ and $\mathrm{HC}_{3} \mathrm{~N}$}

The ratio of abundances, $\mathrm{X}\left(\mathrm{C}_{2} \mathrm{H}\right) / \mathrm{X}\left(\mathrm{HC}_{3} \mathrm{~N}\right)$, indirectly tests the chemical formation path, as $\mathrm{C}_{2} \mathrm{H}$ and $\mathrm{HC}_{3} \mathrm{~N}$ are both thought to involve the same precursor, $\mathrm{C}_{2} \mathrm{H}_{2}$ (Wootten et al. 1980). These authors found the typical ratio to be in the range of 6-10. The most straightforward pathway to the production of $\mathrm{C}_{2} \mathrm{H}$ is through ion-electron recombination with $\mathrm{C}_{2} \mathrm{H}_{2}^{+}$and $\mathrm{C}_{2} \mathrm{H}_{3}^{+}$(Huntress 1977; Herbst, Adams, \& Smith 1987). Among the pathways to produce $\mathrm{HC}_{3} \mathrm{~N}$ is the reaction of $\mathrm{C}_{2} \mathrm{H}_{2}$ with $\mathrm{CN}$ (Herbst \& 

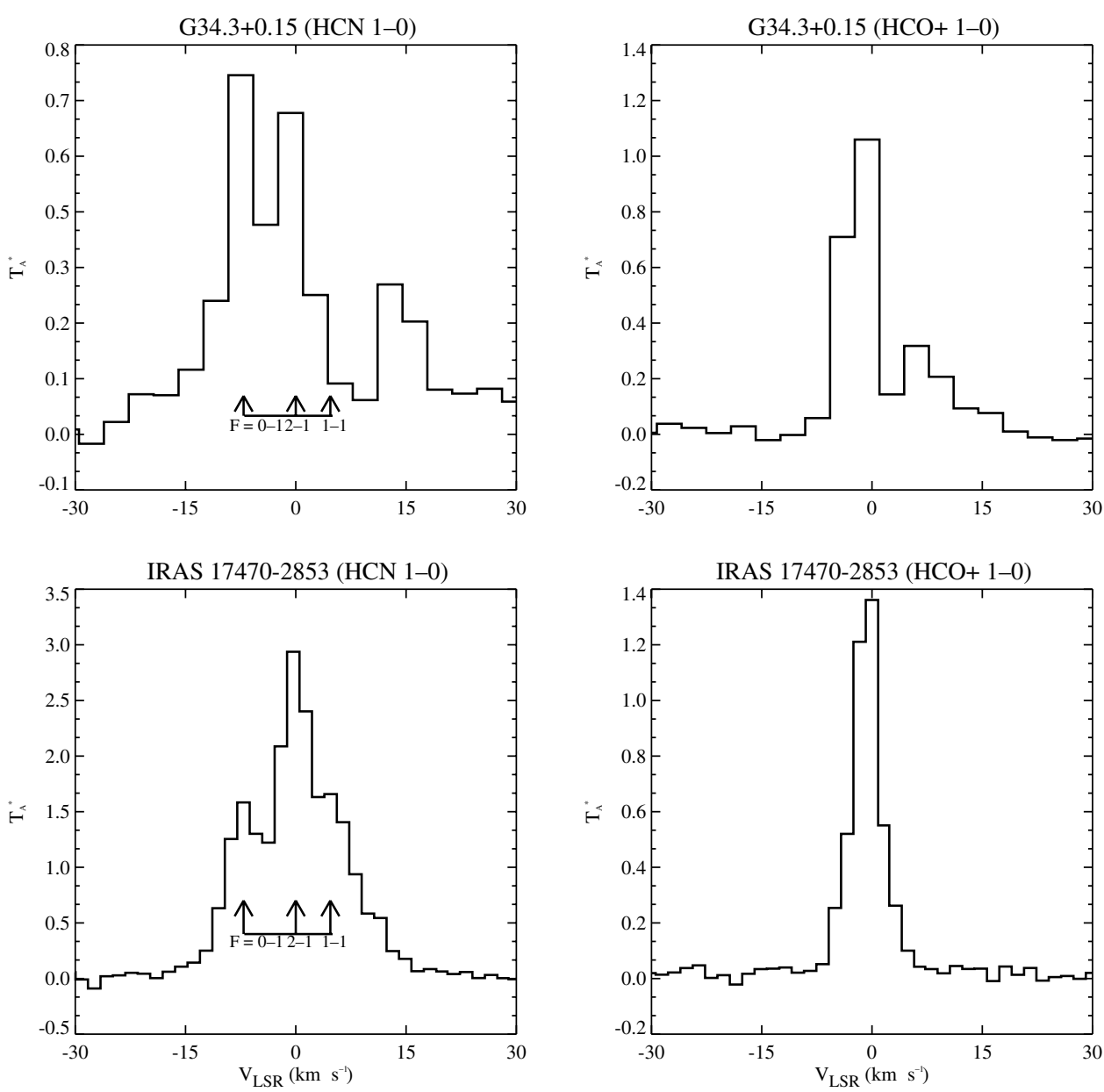

Figure 4 Spectra of $\mathrm{HCN}(1-0)$ and $\mathrm{HCO}^{+}(1-0)$ in G34.3+0.15 (upper panels) and IRAS 17470-2853 (lower panels), as a function of velocity in $\mathrm{km} \mathrm{s}^{-1}$. Positive velocity is in the direction of increasing wavelength. Profiles are plotted with respect to the assumed rest velocity for the sources, $V_{\mathrm{LSR}}=+58 \mathrm{~km} \mathrm{~s}^{-1}$ for G34.3+0.15 and $+14 \mathrm{~km} \mathrm{~s}^{-1}$ for IRAS 17470-2853. The three hyperfine components for $\mathrm{HCN}, F=1-1,2-1$, and $0-1$, are marked.

Table 3. Column densities, optical depths, and excitation temperatures derived for observed molecules

\begin{tabular}{lcccl}
\hline Molecule & $\begin{array}{c}\text { Column density } \\
N_{T}\left(\mathrm{~cm}^{-2}\right)\end{array}$ & $\begin{array}{c}\text { Optical } \\
\text { depth }(\tau)\end{array}$ & $\begin{array}{c}\text { Excitation } \\
\text { temperature, } T_{\mathrm{ex}}(\mathrm{K})\end{array}$ & Notes \\
\hline $\mathrm{HC}_{3} \mathrm{~N}$ & $>3.0 \times 10^{13}$ & & $24^{a}$ & From equation (2) \\
$\mathrm{CH}_{3} \mathrm{CN}$ & $>7.7 \times 10^{13}$ & & $28^{b}$ & From equation (3) \\
$\mathrm{HNC}$ & $>2.7 \times 10^{13}$ & & $4^{a}$ & From equation (2) \\
$\mathrm{C}_{2} \mathrm{H}$ & $>5.7 \times 10^{14}$ & & $8^{a}$ & From equation (2) \\
$\mathrm{SiO}$ & $>1.6 \times 10^{12}$ & & $2^{a}$ & From equation (2) \\
$\mathrm{HCN}$ & $2.4 \times 10^{14}$ & 3.4 & 8 & Equations (6) to (12) \\
$\mathrm{H}^{13} \mathrm{CN}$ & $7.4 \times 10^{12}$ & 0.1 & 8 & \\
$\mathrm{HCO}^{+}$ & $3.5 \times 10^{13}$ & 2.2 & 5 & Equations (6) to (12) \\
$\mathrm{H}^{13} \mathrm{CO}^{+}$ & $5.8 \times 10^{12}$ & 0.4 & 5 & \\
\hline
\end{tabular}

${ }^{a} T_{\mathrm{ex}} \sim T_{\mathrm{rot}}=E_{u} / k$.

${ }^{b} T_{\mathrm{ex}} \sim T_{\mathrm{rot}}=\frac{2}{3} E_{u} / k$.

Leung 1990; Takano et al. 1998). Unfortunately only lower limits for the abundances of $\mathrm{HC}_{3} \mathrm{~N}$ and $\mathrm{C}_{2} \mathrm{H}$ can be derived from our data set (see Section 5.1), so the abundance ratio cannot be well constrained. However, taking these lower limits (see Table 3 ) yields a ratio of $\sim 19$ for $\mathrm{X}\left(\mathrm{C}_{2} \mathrm{H}\right) / \mathrm{X}\left(\mathrm{HC}_{3} \mathrm{~N}\right)$.

\section{$4.3 \mathrm{CH}_{3} \mathrm{CN}$}

This molecule is a symmetric top and all permitted radiative transitions are $\Delta K=0$. Like other symmetric-top molecules, $\mathrm{CH}_{3} \mathrm{CN}$ has been widely used to probe $T_{\text {rot }}$, as it has several closely spaced transitions from a wide range of energy levels that can be observed simultaneously, with 
collisions only responsible for populating the levels along the $K$-ladder. Hence, uncertainties arising from calibration errors and antenna gain can be reduced (Loren \& Mundy 1984). This molecule forms efficiently by the following reaction route (Millar et al. 1991; Mauersberger et al. 1991):

$$
\begin{gathered}
\mathrm{CH}_{3}^{+}+\mathrm{HCN} \rightarrow \mathrm{CH}_{3} \mathrm{CNH}^{+} \\
\mathrm{CH}_{3} \mathrm{CNH}^{+}+\mathrm{e} \rightarrow \mathrm{CH}_{3} \mathrm{CN}+\mathrm{H} .
\end{gathered}
$$

Since $\mathrm{HCN}$ is detected, the possibility of $\mathrm{CH}_{3} \mathrm{CN}$ synthesis by the above reaction route may be high. Two transitions from $\mathrm{CH}_{3} \mathrm{CN}, J=5-4, K=2$ and $K=1$ (the latter blended with the $K=0$ line), are detected. However, the $\mathrm{CH}_{3} \mathrm{CN} J=5-4, K=4$ and $K=3$ lines were not seen in IRAS 17470-2853, whereas they were detected in G34.3+0.15. While this is out of expectation based on the relative line ratios in $\mathrm{G} 34.3+0.16$ (Kim et al. 2000), the $J=5-4, K=2$ measurement is close to the detection limit. The missing lines might simply be lost due to poor signal to noise. ${ }^{2}$

\section{$4.4 \mathrm{SiO}$}

A line from the molecule $\mathrm{SiO}$ was also detected in IRAS 17470-2853. As with G34.3+0.15, the SiO line width is significantly broader than the other lines detected $\left(9 \mathrm{~km} \mathrm{~s}^{-1}\right.$ compared to $\left.4 \mathrm{~km} \mathrm{~s}^{-1}\right)$. This likely reflects a different origin for the $\mathrm{SiO}$ line. Rather than being evaporated off grains in the hot core it is most likely shock-excited, suggesting a mild shock is associated with an embedded source.

\subsection{Lines Not Seen}

For completeness, we finish this section listing some molecules which were not detected in IRAS 17470-2853, but which might have been expected based on comparison with the hot core in Orion-KL or with Sgr B2 (e.g. Turner 1989). However, we also note that none of these molecules was seen in the 86-92 GHz spectral range in G34.3+0.15, either.

No lines from methanol $\left(\mathrm{CH}_{3} \mathrm{OH}\right)$ or sulphur dioxide $\left(\mathrm{SO}_{2}\right)$ were seen, for instance the usually bright lines emitted by methanol at 86.904 and $88.597 \mathrm{GHz}$ and by sulphur dioxide at 86.641 and $91.550 \mathrm{GHz}$. The $\mathrm{HNCO} 4((0,4)-3(0,3))$ line at $87.925 \mathrm{GHz}$ is particularly strong in Orion-KL and Sgr B2, but is absent in IRAS 17470-2853.

\section{Column Densities}

When deriving the excitation temperature and column density of a molecule it is preferable to use several transitions arising from a wide range of energy levels. Fitting for the excitation temperature not only reduces errors from using single lines, but also helps to separate the hot core from

\footnotetext{
${ }^{2}$ In fact, in a deeper spectrum subsequently obtained by us as part of another project, the $K=3$ line is seen with a similar intensity to the $K=2$ line.
}

cooler components in the molecular cloud. The method applied is rotation diagram analysis (see Blake et al. 1987; Macdonald et al. 1996; Kim et al. 2000). This assumes the lines are optically thin and the source is in LTE with a temperature much higher than that of the cosmic background radiation temperature $\left(T_{\mathrm{bg}}=2.7 \mathrm{~K}\right)$. When this is so, the equation of radiative transfer can be simplified so that the rotation temperature and total column density can be derived, as follows:

$$
N_{T}=\frac{3 k \int T_{R}^{*} d v}{8 \pi^{3} v \mu^{2} S g_{I} g_{K}} Q\left(T_{\mathrm{rot}}\right) \exp \left(\frac{E_{u}}{k T_{\mathrm{rot}}}\right),
$$

where $v$ is the transition frequency, $\mu$ the permanent electric dipole moment, $k$ the Boltzmann constant, $g_{I}$ and $g_{K}$ are degeneracies, $E_{u}$ is the upper state energy, $S$ is the line strength, $Q\left(T_{\text {rot }}\right)$ is the partition function for rotational excitation temperature $T_{\text {rot }}$, and $N_{T}$ is the total column density. $\int T_{R}^{*} d v$ is the integrated line intensity. The reduced nuclear spin weight, $g_{I}$, and K-level degeneracy, $g_{K}$, of linear molecules are both 1 . For $\mathrm{CH}_{3} \mathrm{CN}$, however, $g_{K}=1$ for $K=0$ and $g_{K}=2$ for $K \neq 0$, and $g_{I}=1 / 2$ for $K=3 n$ and $g_{I}=1 / 4$ for $K \neq 3 n$ ( $n$ integer) (Turner 1991).

If several lines are observed it is possible to fit a straight line to a plot of $\ln N_{u} / g_{u}=\ln N_{T} / Q \exp \left(-E_{u} / k T\right)$ against $E_{u} / k$, where $N_{u}$ is the upper state column density, to yield the temperature from the slope and the column density from the intercept. However, with our limited spectral coverage, only a single unblended transition of each molecule has been observed (the 91.9853 and $91.9871 \mathrm{GHz}$ lines of $\mathrm{CH}_{3} \mathrm{CN}$ are blended), except for hyperfine transitions of $\mathrm{C}_{2} \mathrm{H}, \mathrm{HCN}$ and $\mathrm{HNC}$. Since these hyperfine lines cover only a small range of upper state energy levels, we considered them as a single line. It is still possible, though, to derive lower limit values from a single line by assuming that the rotation temperature of linear molecules is $T_{\text {rot }}=E_{u} / k$, and for symmetric or slightly asymmetric tops is $T_{\text {rot }}=2 / 3 E_{u} / k$ (see Macdonald et al. 1996). Then, from equation (1), a lower limit to the column density of linear molecules is given by

$$
N_{\min }=\frac{3 k \int T_{R}^{*} d v}{8 \pi^{3} v \mu^{2} S g_{I} g_{K}} Q\left(T_{\text {rot }}\right) e
$$

and for symmetric or slightly asymmetric top molecules by

$$
N_{\min }=\frac{3 k \int T_{R}^{*} d v}{8 \pi^{3} v \mu^{2} S g_{I} g_{K}} Q\left(T_{\mathrm{rot}}\right) e^{3 / 2} .
$$

In the high temperature limit $(h B \ll k T)$ diatomic and linear molecules have a partition function

$$
Q=\sigma \frac{k T}{h B}
$$

where $\sigma=1$ for $\mathrm{HCN}, \mathrm{HNC}, \mathrm{HCO}^{+}$, and $\mathrm{SiO}$, and $\sigma=3$ for $\mathrm{HC}_{3} \mathrm{~N}$ to account for the hyperfine splitting (Blake et al. 1987). The partition function for the symmetric molecule $\mathrm{CH}_{3} \mathrm{CN}$ has the form

$$
Q_{\mathrm{rot}}=\frac{2 \sigma}{3}\left[\pi(k T)^{3} / h^{3} A B^{2}\right]^{1 / 2}
$$


in the high temperature limit $(h A \ll k T, h B \ll k T)$, with $\sigma=3$ to account for hyperfine splitting (Blake et al. 1987).

\section{$5.1 \mathrm{HC}_{3} \mathrm{~N}, \mathrm{CH}_{3} \mathrm{CN}, \mathrm{HNC}, \mathrm{C}_{2} \mathrm{H}$, and $\mathrm{SiO}$ Lower Limits}

Calculating $\int T_{R}^{*} d v$ as $\int T_{A}^{*} d v / \eta_{\mathrm{fss}}$, we then derive $N_{T}>3.0 \times 10^{13} \mathrm{~cm}^{-2}$ for the linear molecule $\mathrm{HC}_{3} \mathrm{~N}$, and $N_{T}>7.7 \times 10^{13} \mathrm{~cm}^{-2}$ for the symmetric molecule $\mathrm{CH}_{3} \mathrm{CN}$. For $\mathrm{HNC}, \mathrm{C}_{2} \mathrm{H}$, and $\mathrm{SiO}$ lower limits of $N_{T}>2.7 \times 10^{13} \mathrm{~cm}^{-2}, N_{T}>5.7 \times 10^{14} \mathrm{~cm}^{-2}$, and $N_{T}>1.6 \times 10^{12} \mathrm{~cm}^{-2}$ are derived, respectively. These limits are listed in Table 3 .

We can compare the limits for $\mathrm{HC}_{3} \mathrm{~N}$ and $\mathrm{CH}_{3} \mathrm{CN}$ with column densities obtained in other surveys. An analysis of data from the NRAO $12 \mathrm{~m}$ telescope for Sgr B2 and Orion-KL (Turner 1991) obtained $N_{T}=3.1 \times$ $10^{14} \mathrm{~cm}^{-2}$ for $\mathrm{HC}_{3} \mathrm{~N}$ and $N_{T}=1.0 \times 10^{14} \mathrm{~cm}^{-2}$ for $\mathrm{CH}_{3} \mathrm{CN}$ in Sgr B2, and $N_{T}=4.6 \times 10^{13} \mathrm{~cm}^{-2}$ for $\mathrm{HC}_{3} \mathrm{~N}$ and $N_{T}=5.6 \times 10^{13} \mathrm{~cm}^{-2}$ for $\mathrm{CH}_{3} \mathrm{CN}$ in Orion-KL. In G34.3+0.15, Kim et al. (2000) obtained $N_{T}=1.1 \times 10^{14}$ $\mathrm{cm}^{-2}$ for $\mathrm{HC}_{3} \mathrm{~N}$ and $N_{T}=1.7 \times 10^{14}$ for $\mathrm{CH}_{3} \mathrm{CN}$. Olmi, Cesaroni, \& Walmsley (1993) observed $\mathrm{CH}_{3} \mathrm{CN}$ towards 11 ultracompact HII regions, finding column densities in the range $2-12 \times 10^{13} \mathrm{~cm}^{-2}$ and rotational temperatures from 8 to $23 \mathrm{~K}$, applying the rotational temperature method.

\section{2 $\mathrm{HCN}$ and $\mathrm{HCO}^{+}$}

For $\mathrm{HCN}$ and $\mathrm{HCO}^{+}$the isotopes $\mathrm{H}^{13} \mathrm{CN}$ and $\mathrm{H}^{13} \mathrm{CO}^{+}$ have also been observed. Assuming the isotopes are optically thin, the main lines are optically thick, and that the levels are in LTE, we can determine the column density, optical depth, and excitation temperature for these molecules as follows:

The equation of radiative transfer is

$$
T_{R}^{*}=\left[J\left(T_{\mathrm{ex}}\right)-J\left(T_{\mathrm{bg}}\right)\right]\left(1-e^{-\tau}\right)
$$

with

$$
J(T)=T_{0}\left[e^{h v / k T}-1\right]^{-1},
$$

where $T_{0}=h \nu / k$. For an optically thick line $(\tau \gg 1)$

$$
T_{\mathrm{ex}}=\frac{T_{0}}{\ln \left(\frac{T_{0}}{T_{R}^{*}+J\left(T_{\mathrm{bg}}\right)}+1\right)} .
$$

On the other hand, if the excitation temperature, $T_{\mathrm{ex}}$, is known, then the optical depth is given by

$$
\tau=-\ln \left(1-\frac{T_{R}^{*}}{J\left(T_{\mathrm{ex}}\right)-J\left(T_{\mathrm{bg}}\right)}\right) .
$$

The optical depth, $\tau$, is related to the column density and excitation temperature by

$$
\tau=\frac{8 \pi^{3} \mu^{2} N}{3 h \Delta v Q}\left[1-e^{-h v / k T_{\mathrm{ex}}}\right] .
$$

From equation (10) we can also relate the optical depth of the main line and the isotope by

$$
\frac{\tau_{\text {main }}}{\tau_{\text {isotope }}} \sim \frac{N_{\text {main }}}{N_{\text {isotope }}}=\mathrm{X},
$$

where $\mathrm{X}$ is the abundance ratio of the main species to its isotope. From equation (6), the ratio of the measured intensities, $R$, is given by

$$
R=\frac{T_{R, \text { main }}^{*}}{T_{R, \text { isotope }}^{*}} \sim \frac{1-e^{-\tau_{\text {main }}}}{1-e^{-\tau_{\text {isotope }}},}
$$

which lets us calculate $\tau_{\text {main }}$. Finally, applying equation (11), and assuming the isotope is optically thin, we obtain

$$
R=\frac{1-e^{-\tau_{\text {main }}}}{\tau_{\text {main }}} \mathrm{X}
$$

Thus, if we assume the main line is optically thick we can derive $T_{\mathrm{ex}}$ from the measured $T_{R}^{*}$ (equation (8)). By assuming the isotope has the same excitation temperature as the main line we derive $\tau_{\text {isotope }}$ (equation (9)). Equation (10) then determines the column density of the isotope, equation (12) the optical depth of the main line, and equation (11) its column density.

For HCN we obtain $T_{\mathrm{ex}} \sim 8 \mathrm{~K}$ and for $\mathrm{H}^{13} \mathrm{CN} \tau \sim 0.1$ and $N_{T} \sim 7.4 \times 10^{12} \mathrm{~cm}^{-2}$. This then determines for $\mathrm{HCN}$ that $\tau \sim 3.4$ and $N_{T} \sim 2.4 \times 10^{14} \mathrm{~cm}^{-2}$.

For $\mathrm{HCO}^{+}$we obtain $T_{\mathrm{ex}} \sim 5 \mathrm{~K}$ and for $\mathrm{H}^{13} \mathrm{CO}^{+} \tau \sim$ 0.4 and $N_{T} \sim 5.8 \times 10^{12} \mathrm{~cm}^{-2}$. This then determines for $\mathrm{HCO}^{+}$that $\tau \sim 2.2$ and $N_{T} \sim 3.5 \times 10^{13} \mathrm{~cm}^{-2}$. These results are summarised in Table 3 .

\section{Summary}

We report the results of the first molecular line survey carried out with the Mopra telescope, towards the massive young stellar source IRAS 17470-2853. Twenty-one molecular transitions from 9 species were detected. We have compared the results to a similar survey for the ultracompact HII region G34.3+0.15.

On the whole, the chemical compositions of IRAS 17470-2853 and G34.3+0.15 are similar. However there are some differences, as might be expected if the sources are at different evolutionary stages. We have found that IRAS 17470-2853 has narrower line widths and weaker antenna temperatures. Self-absorption is not seen in $\mathrm{HCO}^{+}$and the ratio of $\mathrm{HNC} / \mathrm{HCN}$ is significantly lower. There is also a minor difference in the number of lines of $\mathrm{CH}_{3} \mathrm{CN}$ that are detected.

The relatively large line width of $\mathrm{SiO}(v=0, J=$ 2-1) compared to other molecules seen suggests that shock chemistry is occurring, possibly as a result of winds originating from an embedded source.

The isotopic molecules $\mathrm{H}^{13} \mathrm{CN}$ and $\mathrm{H}^{13} \mathrm{CO}^{+}$are seen, as are $\mathrm{C}_{2} \mathrm{H}$ and $\mathrm{HC}_{3} \mathrm{~N}$. These molecules are seen in $\mathrm{G} 34.3+0.15$ too. However, as in $\mathrm{G} 34.3+0.15$, but unlike in the Orion hot core, lines from the molecules $\mathrm{CH}_{3} \mathrm{OH}$, $\mathrm{SO}_{2}$ and $\mathrm{HNCO}$ are not seen from $86-92 \mathrm{GHz}$.

The richness of the spectrum in the relatively small bandpass surveyed suggests that IRAS 17470-2853 will be a fruitful source for further observation, like G34.3+0.15. More comprehensive line surveys of a variety of hot molecular cores, to compare and contrast the molecular 
lines present and their physical characteristics, will provide a tool to study the chemistry of star forming clouds, and the evolutionary stages which they go through as a star is formed. Our data suggest that IRAS 14070-2853 is in a slightly earlier evolutionary stage than G34.3+0.15.

\section{Acknowledgements}

This work would not have been possible without the support of the Australia-Korea Foundation. A number of other people have also made significant contributions to the observational program with the Mopra telescope to whom we are grateful: Tamara Davis, Jung-Kyu Lee, Michael Murphy, Jill Rathborne, Angie Schultz, and John Storey from the University of New South Wales, and Graeme Carrad, Michael Kesteven, and Ray Norris from the Australia Telescope National Facility. We also had useful discussions with Andrew Walsh. Finally, we wish to thank the anonymous referees, whose comments and criticisms have led to a greatly improved paper. The Mopra telescope is operated through a collaborative arrangement between the University of New South Wales and the CSIRO.

\section{References}

Baudry, A., Combes, M., Perault, M., \& Dickman, R. 1980, A\&A, 85,244

Blake, G. A., Sutton, E. C., Masson, C. R., \& Phillips, T. G. 1986, ApJS, 60, 357

Blake, G. A., Sutton, E. C., Masson, C. R., \& Phillips, T. G. 1987, ApJ, 315, 621

Blake, G. A., Mundy, L. G., Carlstrom, J. E., Padin, S., Scott, S. L., Scoville, N. Z., \& Woody, D. P. 1996, ApJ, 472, 49

Brown, R. D., Burden, F. R., \& Cuno, A. 1989, ApJ, 347, 855

Cernicharo, J., Castets, A., Duvert, G., \& Guilloteau, S. 1984, A\&A, 139, L13

Charnley, S. B., Kress, M. E., Tielens, A. G. G. M., \& Millar, T. J. 1995, ApJ, 448, 232

Cummins, S. E., Linke, R. A., \& Thaddeus, P. 1986, APJ, 60, 819

Forster, J. R., \& Caswell, J. L. 1999, A\&AS, 137, 43

Garwood, R. W., \& McMullin, J. P. 1999, AIPS++ NOTE 225Using Dish: The AIPS ++ Single Dish Analysis Environment, http://aips2.nrao.edu/docs/aips++.html
Goldsmith, F. P., Langer, W. D., Ellder, J., Irvine, W., \& Kollberg, E. 1981, ApJ, 249, 524

Henkel, C., Mauersberger, R., Wilson, T. L., Snyder, L. E., Menten, K., \& Wouterloot, J. G. A. 1987, A\&A, 182, 299

Herbst, E., Adams, N. G., \& Smith, D. 1987, ApJ, 312, 351

Herbst, E., \& Leung, C. M. 1990, A\&A, 233, 177

Huntress, W. T. 1977, ApJS, 33, 495

Huttemeister, S., Henkel, C., Mauersberger, R., Brouillet, N., Wiklind, T., \& Millar, T. J. 1995, A\&A, 295, 571

Irvine, W. I., \& Schloerb, F. P. 1984, ApJ, 282, 516

Jewell, P. R., Hollis, J. M., Lovas, F. J., \& Snyder, L. E. 1989, ApJS, 70,833

Johansson, L. E. B., et al. 1984, A\&A, 130, 227

Kim, H.-D., Cho, S.-H., Chung, H.-S., Kim, H.-R., Roh, D.-G., Kim, H.-G., Minh, Y. C., \& Minn, Y.-K. 2000, ApJS, 131, 483

Loren, R. B., \& Mundy, L. G. 1984, ApJ, 286, 232

Lovas, F. J. 1992, JPCRD, 21, 181

Macdonald, G. H., Gibb, A. G., Harbing, R. J., \& Millar, T. J. 1996, A\&AS, 119, 333

Mauersberger, R., Henkel, C., Walmsley, C. M., Sage, L. J., \& Wiklind, T. 1991, A\&A, 247, 307

Millar, T. J., Herbst, E., \& Charnley, S. B. 1991, A\&A, 369, 147

Millar, T. J., Macdonald, G. H., \& Gibb, A. G. 1997, A\&A, 325, 1163

Olmi, L., Cesaroni, R., \& Walmsley, C. M. 1993, A\&A, 276, 489

Schilke, P., Walmsley, C. M., Pineau des Forets, G., Roueff, E., Flower, D. R., \& Guilloteau, G. 1992, A\&A, 256, 595

Schilke, P., Groesbeck, T. D., Blake, G. A., \& Phillips, T. G. 1997, ApJS, 108, 301

Sutton, E. C., Jaminet, P. A., Danchi, W. C., \& Blake, G. A. 1991, ApJS, 77, 255

Takano, S., et al. 1998, A\&A, 329, 1156

Turner, B. E. 1989, ApJS, 70, 539

Turner, B. E. 1991, ApJS, 76, 617

Ulich, B. L., \& Haas, R. W. 1976, ApJS, 30, 247

Walmsley, C. M., Hersen, W., Henkel, C., Mauersberger, R., \& Wilson, T. L. 1987, A\&A, 172, 311

Walsh, A. J., Hyland, A. R., Robinson, G., \& Burton, M. G. 1997, A\&A, 291, 261

Walsh, A. J., Burton, M. G., Hyland, A. R., \& Robinson, G. 1998, A\&A, 301, 640

Walsh, A. J., Macdonald, G. H., Alvey, N. P. S., Burton, M. G., \& Lee, J.-K. 2002, A\&A, submitted

Wootten, A., Evans II, N. J., Snell, R., \& Vanden Bout, P. 1978, ApJ, 225, L143

Wootten, A., Bozyan, E. P., \& Garrett, D. B. 1980, ApJ, 239, 844

Ziurys, L. M., \& McGonagle, D. 1993, ApJS, 89, 155 